\title{
Capsule Commentary on Chiu et al. BMI Trajectories as a Harbinger of Pre-Diabetes or Underdiagnosed Diabetes: An 18-Year Retrospective Cohort Study in Taiwan
}

\author{
Rupam Ruchi, MD \\ University of Florida, Gainesville, FL, USA. \\ J Gen Intern Med 31(10): 1216 \\ DOI: $10.1007 / \mathrm{s} 11606-016-3777-0$ \\ (c) Society of General Internal Medicine 2016
}

$\mathrm{T}$ he study by Chiu et al. ${ }^{1}$ comparing BMI trajectories between diabetics and non-diabetics is particularly relevant to Asia. The incidence of diabetes is increasing alarmingly throughout the continent. Genetic differences combined with recent changes in dietary habits in these nations have caused an epidemic of diabetes. ${ }^{2}$

Type 2 diabetes is notoriously "silent." Many patients will not develop overt symptoms until they develop complications, which might be too late. In one study, approximately $20 \%$ of diabetics in South Asian countries did not know they had diabetes. ${ }^{3}$

This study found that the baseline weight of Asians who develop diabetes is significantly higher than that of those who do not, and the amount of weight gained is greater. The strength of the study lies in the long follow-up before the diagnosis of diabetes and comparison with those who did not develop diabetes. Interestingly, they noted a drop in weight $\sim 6.5$ years before diagnosis, a finding never previously reported. The biological basis for this is uncertain. The authors cite the lack of availability of glucose as fuel, prompting the tissues to break down muscle cells for gluconeogenesis; its occurrence 6.5 years before diagnosis makes this somewhat of a stretch. A prospective study of 1553 Japanese non-diabetic men, followed annually for 10 years by anthropometric and laboratory tests, did not show any significant weight loss in those who developed diabetes after 10 years. ${ }^{4}$ It is hard to know whether this is a real finding or a statistical anomaly. Moreover, the amount of weight loss noted, about $1.25 \mathrm{~kg}$, is not very noticeable in someone with an increased BMI. Hence, practical implications of this finding are limited by the minimal weight change. Nevertheless, the study lays the groundwork for future trials to investigate its clinical relevance and the biological basis of this weight loss specifically in the middle-aged Asian population.

Corresponding Author: Rupam Ruchi, MD; University of Florida, Gainesville, FL, USA (e-mail: rupam.ruchi@medicine.ufl.edu).

\section{Compliance with Ethical Standards:}

Conflict of Interest: The author has no conflicts of interest with this article.

\section{REFERENCES}

1. Chiu CJ, Li SL, Wu CH, Du YF. BMI trajectories as a harbinger of prediabetes or underdiagnosed diabetes: an 18-year retrospective cohort study in Taiwan. J Gen Intern Med. 2016. doi:10.1007/s11606-0163750-y.

2. Hu FB. Globalization of diabetes: the role of diet, lifestyle, and genes. Diabetes Care. 2011;34(6):1249-57.

3. Carrillo-Larco RM, Miranda JJ, Li X, Cui C, Xu X, et al. Prevalence of pragmatically defined high CV risk and its correlates in LMIC: a report from 10 LMIC areas in Africa, Asia, and South America. Glob Heart. 2016;11(1):27-36.

4. Heianza Y, Arase Y, Kodama S, et al. Trajectory of body mass index before the development of type 2 diabetes in Japanese men: Toranomon Hospital Health Management Center Study 15. J Diabetes Investig. 2015;6(3):28994.

Published online July 11, 2016 